\title{
Synthesis of some New Schiff Bases Containing Acridone Moiety
}

\author{
Attalla M. Sheat \\ Department of Chemistry/ College of Science/ University of Mosul \\ E-mail: attalsharifi@yahoo.com
}

(Received 16/1/2018; Accepted 3/5/2018)

\begin{abstract}
The 2-[(4'-amino-3,3'-dimethylbiphenyl-4-yl)amino]benzoic acid (II) was synthesized by Ullmann-Goldberg coupling by reaction of 3,3-dimethylbiphenyl-4,4-diamine (I) with 2chlorobenzoic acid. The compound (II) was cyclized by poly phosphoric acid (PPA) to give 2-[(4amino-3-methyl)phenyl]-4-methylacridin-9 (10H)-one (III). New Schiff bases (IVa-j) and (Va-d) has been prepared by reaction of compound (III) with aromatic aldehydes and cyclic ketones respectively.
\end{abstract}

Keywords: Schiff bases, Acridone moiety.

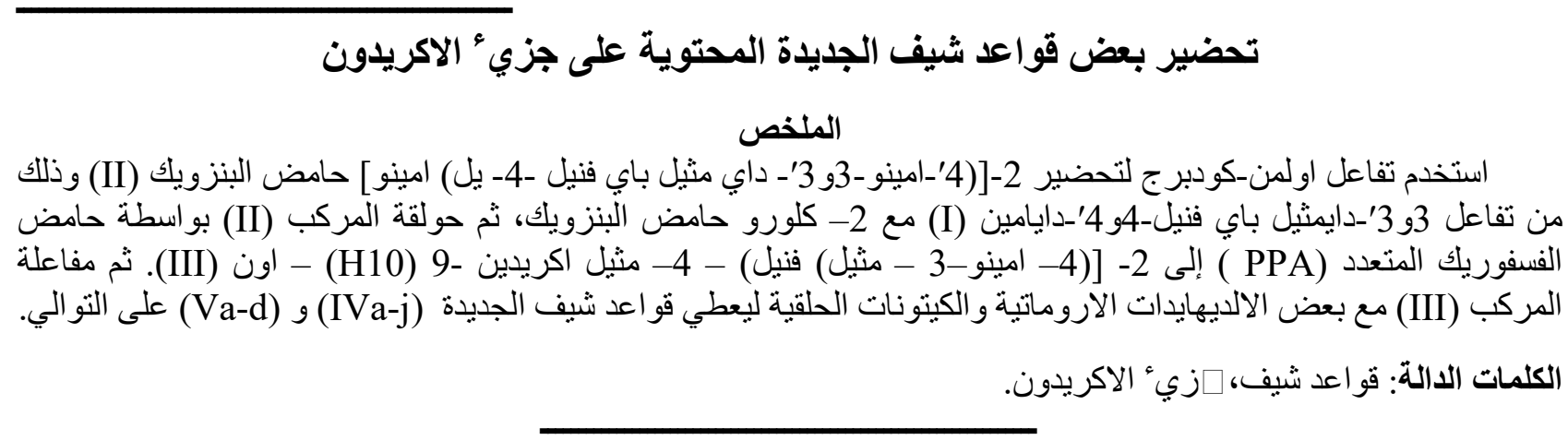

\section{INTRODUCTION}

The basic tricyclic framework can be decorated with suitable substituents to confer specificity against both prokaryotic and eukaryotic targets which have given acridines a respectable reputation in the history of chemotherapy (Velingker and Dandekar, 2009). Fused heterocyclic acridone is one of scaffolds known to be associated with biological activities due to the pharmacological activities of its nucleus. It has a carbonyl group and nitrogen atom at 9 and 10 position respectively.

Acridones of natural and synthetic origins are known to possess a wide variety of biological activities (Ajala and Okoro, 2012), including inhibitory action against viruses (Goodell et al., 2006), and used as anticancer (Amareswarao et al., 2016, Kandeel et al., 2016), anti-tumor (Huang et al., 2015), anti-leukemia (Wang, et al., 2013), anticonvulsant (Mohammad et al., 2016), antimicrobial (Kadryavtsera et al., 2015; Gupta et al., 2015), anti-malarial (Valdes, 2011), anti-proliferative (Ugarte et al., 2012), and it also used as acetylcholinesterase inhibitor (Khanposhtani et al., 2015).

\section{EXPERIMENTAL}

Melting point was determined using Electro thermal IA9000 Digital-series melting point apparatus, (uncorrected). All reagents and chemicals were used from commercial sources. The solvents were dried by standard procedures. The purity of the compounds was ascertained by thin layer chromatography (TLC) on pre-coated silica gel glass plates using either UV radiation or iodine staining for visualization. Column chromatography was carried out using 100-200 mesh 
silica gel (BDH). IR spectra were recorded using FT-IR-600 Bio Tech Engineering Management spectrophotometer UK using $\mathrm{KBr}$ disc. ${ }^{1} \mathrm{H}-\mathrm{NMR}$ and ${ }^{13} \mathrm{C}-\mathrm{NMR}$ spectra were obtained from a Bruker-avance $300 \mathrm{MHz}$, NMR spectrometer. The chemical shifts are reported as $\delta$ ( ppm ) for the DMSO- $\mathrm{d}_{6}$ solution using TMS as internal standard. The coupling constant, $\mathrm{J}(\mathrm{Hz})$ in that order with the use of the following abbreviations; s, singlet; d, doublet; t, triplet; m, multiplet; br, broad.

Synthesis of 2[(4'-amino-3,3'-dimethylbiphenyl-4-yl)amino]benzoic acid (II) (Mei et al., 2006; Wolf and Mei, 2003).

A mixture of 3,3'-dimethylbiphenyl-4,4'-diamine (I) (25 mmol, $5.3 \mathrm{~g}$ ), 2-chlorobenzoic acid (24 mmol, $3.8 \mathrm{~g})$, anhydrous $\mathrm{K}_{2} \mathrm{CO}_{3}(30 \mathrm{mmol}, 4.1 \mathrm{~g})$, $\mathrm{Cu}$ powder $(0.05 \mathrm{~g})$, and $\mathrm{Cu}_{2} \mathrm{O}(0.05 \mathrm{~g})$ in $5 \mathrm{ml}$ of 2-ethoxyethanol was refluxed at $130{ }^{\circ} \mathrm{C}$ for 3 hours. The cooled reaction mixture was poured into $30 \mathrm{ml}$ of water. Charcoal was then added and the solution was filtered through celite. The crude product was obtained by a precipitation. Acidification of the filtrate with diluted $\mathrm{HCl}$, and subsequent recrystallization from acetone/water (1:8). The crystals were dissolved in $100 \mathrm{ml}$ of 5\% aqueous $\mathrm{Na}_{2} \mathrm{CO}_{3}$. The solution was filtered through a celite and the product was precipitated by acidification of the filtrate to afford acid (II), $(6.18 \mathrm{~g}, 55 \%)$, as a green powder (m.p $=229-231$ $\left.{ }^{\circ} \mathrm{C}\right),\left(\mathrm{R}_{\mathrm{f}}=0.55\right)$ (chloroform: methanol 9.5: 0.5).

Synthesis of 2-(4-amino-3-methylphenyl)-4-methylacridin-9(10H)-one (III) (Denny et al., 1977; Jameel and Sheat, 2014)

The acid (II) (5g) was heated with polyphosphoric acid (PPA) $(50 \mathrm{ml})$ at $120-130{ }^{\circ} \mathrm{C}$ until a homogeneous solution resulted. The heating was continued for $3 \mathrm{hrs}$. The product was precipitated by addition of $\mathrm{H}_{2} \mathrm{O}$ and basification with $\mathrm{NH}_{4} \mathrm{OH}$. The solid material was filtered off by vacuum and washed several times with water then air dried to give $(4.1 \mathrm{~g}, 87 \%)$, as green powder (m.p $\left.>325^{\circ} \mathrm{C} \mathrm{dec}\right),\left(\mathrm{R}_{\mathrm{f}}=0.67\right)$ (chloroform: methanol 9.5: 0.5).

\section{Synthesis of 2-[4-(benzylidineamino)-3-methylphenyl]-4-methylacridin-9(10H)-one(IVb-j),} (Va-d) (Kannappan et al., 2009; Belwal and Joshi, 2012) (General procedure)

To a solution of compound III $(0.2 \mathrm{~g}, 0.63 \mathrm{mmol})$ in dry methanol $(25 \mathrm{ml})$, benzaldehyde $(0.06 \mathrm{~g}, 0.63 \mathrm{mmol})$ was added. The resulted solution was refluxed with stirring for at least 6 hours. The proceeding of the reaction was monitored by TLC. The mixture was cooled and left overnight. The solid product was filtered off and dried in air $\left(40 \%, \mathrm{~m} . \mathrm{p}=275^{\circ} \mathrm{C}\right)$. The same procedure was used to prepare the compounds (IVb-j) and (Va-d) by mixing of ( $0.63 \mathrm{mmol}$ of compound (III) with appropriate substituted benzaldehyde and cyclic ketones. Table (1) summarizes the physical properties and spectral data for compounds $(\mathrm{IVa}-\mathrm{j})$ and $(\mathrm{Va}-\mathrm{d}) \cdot\left(\mathrm{R}_{\mathrm{f}}=\right.$ chloroform: methanol 9.5: $0.5)$. 
Table 1: The Physical properties and the FT-IR spectral data (in $\mathrm{cm}^{-1}$ ) for compounds (IVa-j) and (Va-d)

\begin{tabular}{|c|c|c|c|c|c|c|c|c|c|}
\hline Compd. No. & $\mathbf{X}$ & m.p. ${ }^{0} \mathrm{C}$ & $\mathbf{R}_{\mathbf{f}}$ & color & $\begin{array}{c}\text { Yield } \\
\%\end{array}$ & N-H Str. & $\begin{array}{l}C=\mathbf{O} \\
\text { Str. }\end{array}$ & $\begin{array}{l}C=N \\
\text { Str. }\end{array}$ & $\begin{array}{l}C=C \\
\text { Str. }\end{array}$ \\
\hline Iva & $\mathrm{H}$ & 275 & 0.82 & $\begin{array}{l}\text { Greenish- } \\
\text { yellow }\end{array}$ & 40 & $3307-3202$ & 1623 & 1591 & 1571 \\
\hline $\mathrm{IVb}$ & $4-\mathrm{Br}$ & 305 & 0.8 & Green & 55 & $3335-3260$ & 1622 & 1590 & 1568 \\
\hline IVc & $3-\mathrm{NO}_{2}$ & $310-312$ & 0.67 & $\begin{array}{l}\text { Reddish- } \\
\text { Brown }\end{array}$ & 57 & $3355-3248$ & 1625 & 1595 & 1568 \\
\hline $\mathrm{IVd}$ & $4-\mathrm{NO}_{2}$ & $320-322$ & 0.65 & Red & 60 & $3336-3215$ & 1630 & 1602 & 1565 \\
\hline IVe & 2-OH & $300-302$ & 0.64 & Yellow & 35 & $3365-3210$ & 1633 & 1605 & 1567 \\
\hline IVf & $\begin{array}{c}4-\mathrm{OH} \\
, 3-\mathrm{OCH}_{3}\end{array}$ & $270-272$ & 0.63 & Yellow & 32 & $3332-3277$ & 1625 & 1595 & 1561 \\
\hline $\mathrm{IVg}$ & $4-\mathrm{OCH}_{3}$ & $265-267$ & 0.71 & Yellow & 35 & $3368-3313$ & 1627 & 1603 & 1571 \\
\hline IVh & $\begin{array}{c}4- \\
\mathrm{N}\left(\mathrm{CH}_{3}\right)_{2}\end{array}$ & 306-308 & 0.75 & $\begin{array}{l}\text { Yellowish } \\
\text {-Black }\end{array}$ & 30 & $3350-3221$ & 1635 & 1601 & 1565 \\
\hline IVi & 2-COOH & $282-284$ & 0.59 & Brown & 69 & $3361-3232$ & 1623 & 1598 & 1567 \\
\hline $\mathrm{IVj}$ & 4- $\mathrm{Cl}$ & $258-260$ & 0.78 & Yellow & 72 & $3380-3227$ & 1638 & 1593 & 1571 \\
\hline $\mathrm{Va}$ & & $142-144$ & 0.66 & Black & 35 & $3370-3225$ & 1625 & 1591 & 1572 \\
\hline $\mathrm{Vb}$ & & $120-122$ & 0.69 & Brown & 40 & $3375-3226$ & 1625 & 1601 & 1595 \\
\hline $\mathrm{Vc}$ & & $90(d)$ & 0.63 & Brown & 38 & $3378-3220$ & 1623 & 1595 & 1575 \\
\hline $\mathrm{Vd}$ & & $170(d)$ & 0.61 & Brown & 35 & $3360-3215$ & 1630 & 1597 & 1572 \\
\hline
\end{tabular}

d. decomposition

\section{RESULTS AND DISCUSSION}

\section{Synthesis of 2[(4'-amino-3,3'-dimethylbiphenyl-4-yl)amino]benzoic acid (II).}

$2\left[\left(4^{\prime}\right.\right.$-Amino-3,3'-dimethylbiphenyl-4-yl)amino]benzoic acid (II) was prepared by mixing 3,3'dimethylbiphenyl-4,4'-diamine (I) with 2-chlorobenzoic acid, in presence anhydrous potassium carbonate as a base, 2- ethoxy ethanol as a solvent, cuprous oxide and copper metal as a catalyst as shown in the following equation.<smiles>Cc1cc(-c2ccc(N)c(C)c2)ccc1N</smiles>

(I)

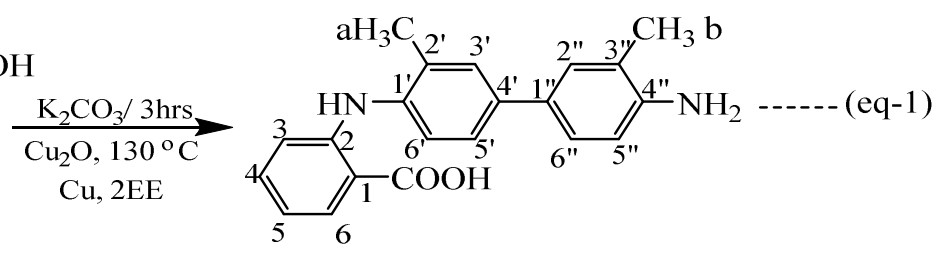

(II) 
The structure of the acid(II) was identified by FT-I.R, ${ }^{1} \mathrm{H}-\mathrm{NMR}$, and ${ }^{13} \mathrm{C}-\mathrm{NMR}$ spectral data. The IR spectra showed the following main absorption bands $\left(\mathrm{cm}^{-1}\right)$ at: $1598(\mathrm{C}=\mathrm{C}), 1655(\mathrm{C}=\mathrm{O})$, 3310-2350 (NH, NH$), 3460(\mathrm{OH})$ (Jameel and Mohammed, 2011). ${ }^{1} \mathrm{H}-\mathrm{NMR}$ spectrum showed the following chemical shifts $(\delta, \mathrm{ppm}) . \delta=2.12\left(\mathrm{~s}, \mathrm{CH}_{3} \mathrm{~b}\right), 2.23\left(\mathrm{~s}, \mathrm{CH}_{3}\right.$ a), 3.37 (br, $\left.\mathrm{NH}_{2}\right), 6.66-6.91$ $(\mathrm{m}, 11 \mathrm{H}, \mathrm{Ar}-\mathrm{H}, \mathrm{N}-\mathrm{H}), 9.52(\mathrm{br}, 1 \mathrm{H}, \mathrm{OH}) .{ }^{13} \mathrm{C}-\mathrm{NMR} \delta=18.09\left(\mathrm{CH}_{3} \mathrm{~b}\right), 18.30\left(\mathrm{CH}_{3} \mathrm{a}\right), 112\left(\mathrm{C}_{1}\right)$, $113.72\left(\mathrm{C}_{5^{\prime \prime}}\right), 114.78\left(\mathrm{C}_{5}\right), 116.94\left(\mathrm{C}_{3}\right), 121.81\left(\mathrm{C}_{3^{\prime \prime}}\right), 123.78\left(\mathrm{C}_{4^{\prime}}\right), 124.15\left(\mathrm{C}_{5^{\prime}}\right), 124.90\left(\mathrm{C}_{6^{\prime \prime}}\right), 127.91$ $\left(\mathrm{C}_{6}\right), 128.38\left(\mathrm{C}_{1^{\prime \prime}}\right), 128.38\left(\mathrm{C}_{4^{\prime}}\right), 132.26\left(\mathrm{C}_{3^{\prime}}\right), 132.26\left(\mathrm{C}_{2^{\prime \prime}}\right), 134.60\left(\mathrm{C}_{2^{\prime}}\right), 136.29\left(\mathrm{C}_{4}\right), 137.29\left(\mathrm{C}_{1^{\prime}}\right)$, $146.53\left(\mathrm{C}_{2}\right), 148.63\left(\mathrm{C}_{4 "}\right), 170.75(\mathrm{C}=\mathrm{O})$. (Girohar et al., 2010).

\section{Synthesis of 2-(4-amino-3-methylphenyl)-4-methylacridin-9(10H)-one (III).}

The compound (III) was prepared by cyclization of the acid (II) using (PPA) according to the following equation. The mechanism of this reaction (scheme 1) involves cyclodehydration of the corresponding acid (Ganan, 1973).

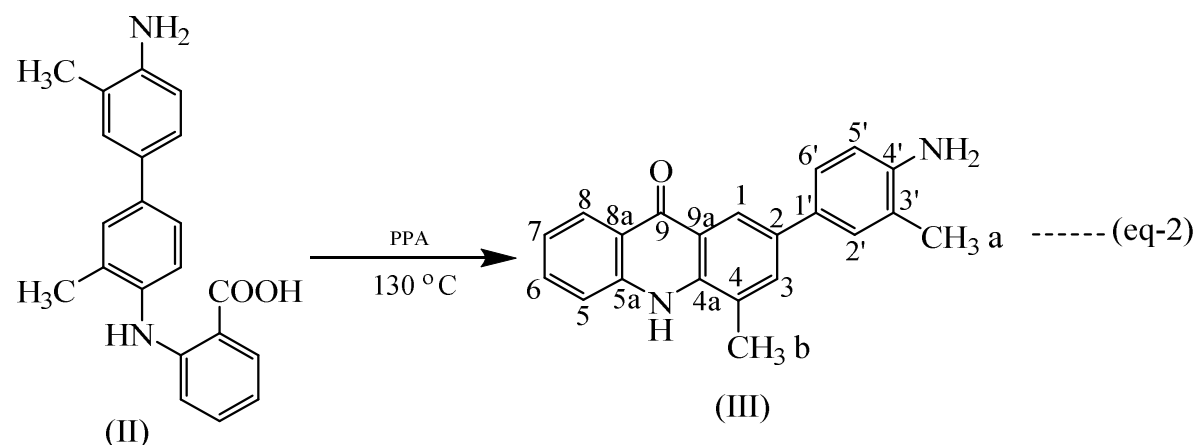

(II)<smiles>Cc1cc(-c2ccc(Nc3ccccc3C(=O)O)c(C)c2)ccc1N</smiles>

(II)<smiles>Cc1cc(-c2ccc(Nc3ccccc3C(=O)O)c(C)c2)ccc1N</smiles><smiles>Cc1cc(-c2cc(C)c3[nH]c4ccccc4c(=O)c3c2)ccc1N</smiles>

Scheme (1)

The structure of compound (III) was identified by FT- I.R , ${ }^{1} \mathrm{H}-\mathrm{NMR}$, and ${ }^{13} \mathrm{C}-\mathrm{NMR}$ spectral data. The IR spectra showed the following main absorption bands $\left(\mathrm{cm}^{-1}\right)$ at $1590(\mathrm{C}=\mathrm{C}), 1623$ $(\mathrm{C}=\mathrm{O}), 3340-3250\left(\mathrm{NH}, \mathrm{NH}_{2}\right)$ (Ganan, 1973). ${ }^{1} \mathrm{H}-\mathrm{NMR}$ spectrum showed the following chemical shifts $(\delta, \mathrm{ppm}) . \delta=2.15\left(\mathrm{~s}, \mathrm{CH}_{3} \mathrm{a}\right), 2.64\left(\mathrm{~s}, \mathrm{CH}_{3} \mathrm{~b}\right), 3.49\left(\mathrm{~s}, \mathrm{NH}_{2}\right), 6.71-8.32(\mathrm{~m}, 9 \mathrm{H}, \mathrm{Ar}-\mathrm{H}), 10.66$ $(\mathrm{s}, 1 \mathrm{H}, \mathrm{NH}) .{ }^{13} \mathrm{C}-\mathrm{NMR} \delta=18.09\left(\mathrm{CH}_{3} \mathrm{~b}\right), 18.44\left(\mathrm{CH}_{3} \mathrm{a}\right), 114\left(\mathrm{C}_{5^{\prime}}\right), 118.65\left(\mathrm{C}_{5}\right), 119.63\left(\mathrm{C}_{8 \mathrm{a}}\right), 119.63$ $\left(\mathrm{C}_{9 \mathrm{a}}\right), 120.64\left(\mathrm{C}_{4}\right), 121.59\left(\mathrm{C}_{7}\right), 122.00\left(\mathrm{C}_{3^{\prime}}\right), 124.99\left(\mathrm{C}_{1}\right), 126.25\left(\mathrm{C}_{2^{\prime}}\right), 126.25\left(\mathrm{C}_{6^{\prime}}\right), 127.48\left(\mathrm{C}_{8}\right)$, $128.38\left(\mathrm{C}_{3}\right), 132.60\left(\mathrm{C}_{6}\right), 133.52\left(\mathrm{C}_{2}\right), 134.27\left(\mathrm{C}_{1^{\prime}}\right), 141.37\left(\mathrm{C}_{5 \mathrm{a}}\right), 143.27\left(\mathrm{C}_{4 \mathrm{a}}\right), 146.67\left(\mathrm{C}_{4}\right), 177.60$ (C) (Avellaneda et al., 2002). 


\section{Synthesis of 2-[4-(substituted-benzylidineamino)-3-methylphenyl]-4-methyl acridin-9(10H)- one (IVa-j).}

The compounds (IVa-j) were prepared by heating an equimolar of compound (III) with aromatic aldehydes in dry methanol according to the following equation.

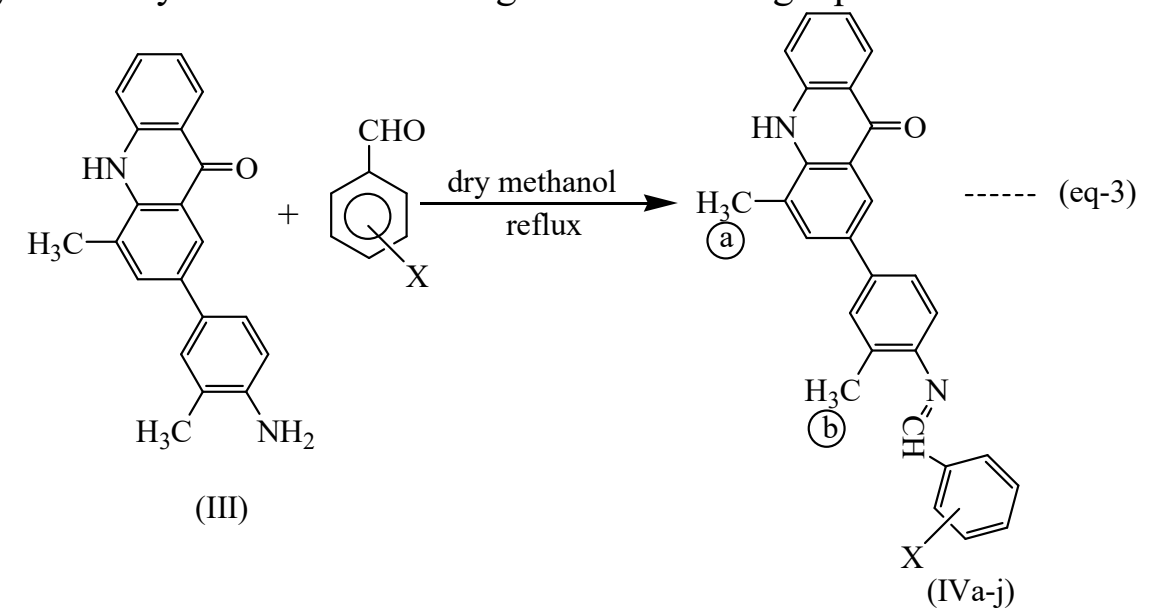

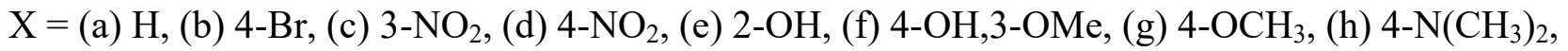

(i) $2-\mathrm{COOH},(\mathrm{j}) 4-\mathrm{Cl}$

The structures of these compounds were identified by FT-I.R and ${ }^{1} \mathrm{H}-\mathrm{NMR}$ spectral data; IR spectra showed a main absorption bands $\left(\mathrm{cm}^{-1}\right)$ Table $(1)$ at: $3380-3215(\mathrm{~N}-\mathrm{H}), 1638-1622(\mathrm{C}=\mathrm{O})$, 1605-1591 $(\mathrm{C}=\mathrm{N})$, 1565-1571 $(\mathrm{C}=\mathrm{C})$ (Hodgeman and Prager, 1971). ${ }^{1} \mathrm{H}-\mathrm{NMR}$ spectrum for compound (IVd) showed the following chemical shifts $(\delta, \mathrm{ppm}) . \quad \delta=2.47\left(\mathrm{~s}, \mathrm{CH}_{3} \mathrm{a}\right), 2.72$ $\left(\mathrm{s}, \mathrm{CH}_{3} \mathrm{~b}\right), 7.28-8.41(\mathrm{~m}, 13 \mathrm{H}, \mathrm{Ar}-\mathrm{H}),(\mathrm{s}, 1 \mathrm{H}, \mathrm{HC}=\mathrm{N}), 10.74(\mathrm{~s}, 1 \mathrm{H}, \mathrm{NH}) .{ }^{1} \mathrm{H}-\mathrm{NMR}$ spectrum for compound (IVi) showed the following chemical shifts $(\delta, \mathrm{ppm}): \delta=2.75\left(\mathrm{~s}, \mathrm{CH}_{3} \mathrm{~b}\right), 2.68\left(\mathrm{~s}, \mathrm{CH}_{3} \mathrm{a}\right)$, $6.70(\mathrm{~d}, 1 \mathrm{H}, \mathrm{J}=7.2 \mathrm{~Hz}), 6.70-8.25(\mathrm{~m}, 14 \mathrm{H}, \mathrm{Ar}-\mathrm{H}, \mathrm{COOH}), 8.33(\mathrm{~s}, 1 \mathrm{H}, \mathrm{HC}=\mathrm{N}), 10.71(\mathrm{~s}, 1 \mathrm{H}$, NH). (Drogon et al., 2010; Gao et al., 2012).

\section{Synthesis of 2-[4-(cycloalkylidineamino)-3-methylphenyl]-4-methylacridin-9(10H)-one (Va-d).}

These compounds were prepared by heating of equimolar of compound (III) with various cycloketones in dry methanol as shown in the following equation.

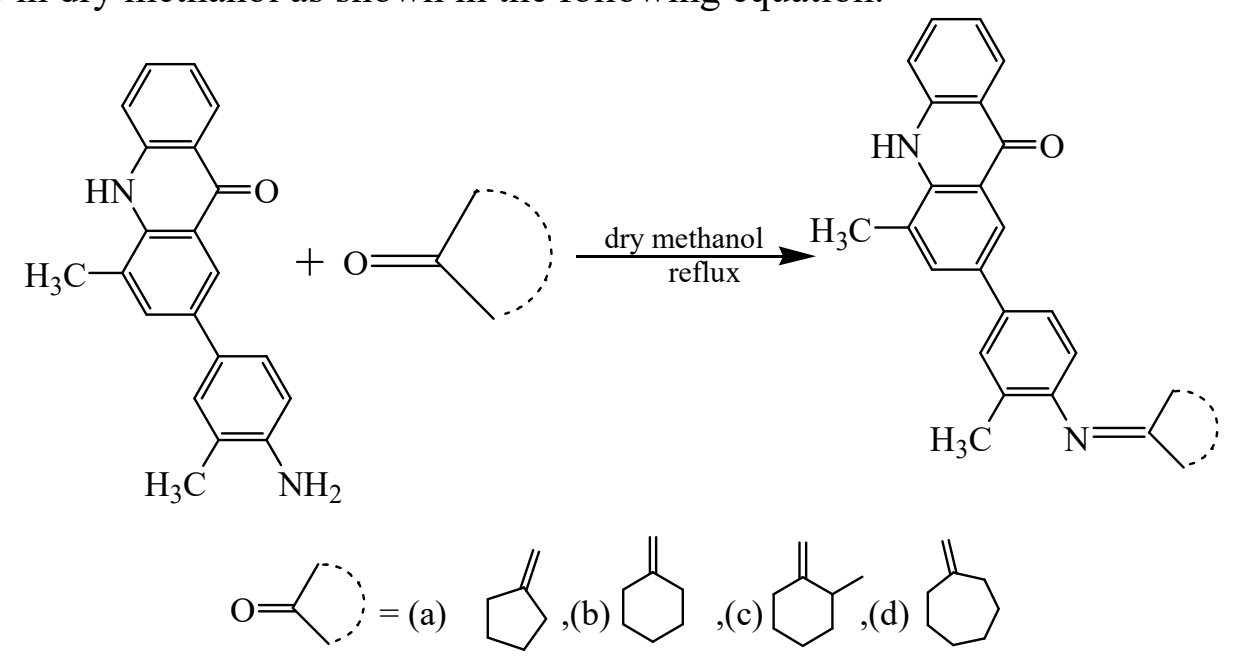

The structures of these compounds (Va-d) were identified by the I.R spectral data. The I.R spectra of these compounds showed a main absorption bands $\left(\mathrm{cm}^{-1}\right)$, Table (1) at 3378-3215 (N-H), 1623-1630 $(\mathrm{C}=\mathrm{O}), 1591-1601(\mathrm{C}=\mathrm{N}), 1595-1672(\mathrm{C}=\mathrm{C})$. 


\section{ACKNOWLEDGEMENTS}

The author is thankful to the head of Chem. Dept. and to the dean of Sci. Coll., Univ. of Mosul for providing finance for spectral measurements.

\section{REFERENCES}

Ajala, A.O.; Okoro, C.O.; (2012). QSAR Topomer Co MFA studies on 10 N- substituted acridone derivatives. Open J. Medicinal Chemistry., 2, 43-49.

Amareswarao, M.; Babu, Y.R.; Himabindhu, J.; Rajendra, V.V.S. (2016). Synthesis of nitric donating acridone derivatives as cytotoxic agents in cancer. Der Pharma Chemica., 8(4), 391-398.

Avellaneda, A.; Robin, M.; Feure, R.; Perichaud, A.; Galy, J.P. (2002). Spectral assignment and reference data. Mag. Res .Chem., 40, 545-548.

Belwal, C.K.; Joshi, K.A. (2012). Synthesis and antifungal activity of some novel thiazolidinone derivatives of 4-(4-oxo-2-phenylthiazolidin-3-yl)benzoic acid. Int. J. Chem. Tech. Res., 4(4), $1758-1764$.

Denny, W.A.; Atwell, G.J.; Cain, B.F. (1977). Potential anti-tumor agents. 25. Azalogues of the 4'(9-acridinylamino) methane sulfonanilides. J. Med .Chem., 20(10), 1242.

Drogon, A.S.; Dornor. B.; Thomas, E.; Boguszewsk-Chachulska, M. (2010). Synthesis of new acridone derivatives, inhibitors of NS3 helicase, which efficiently and specifically inhibit sub genomic HCV replication. J. Med. Chem., 53, 3117-3126.

Ganan, J.M.F. (1973). "Chemistry of Heterocyclic Compounds". Volume 9, John Wiley and Sons, Inc., England, pp. 141-377.

Girohar, A.; Jain, S.; Jain, N.; Girdha, S. (2010). Synthesis and biological studies of novel 9(10H)acridone derivatives. Acta Poloniae-Drug Research.,67(2), 211-214.

Goa, Ch.; Li, Sh.; Lang, X.; Liu, H.; Liu, F.; Tan, Ch.; Jiang, Y. (2012). Synthesis and evaluation of 10-(3,5-dimethoxy)benzyl-9(10H)-acridone derivatives as selective telomeric Gquadruplex DNA ligans. Tetrahedrone., 68, 7920-7925.

Gooldell, J.R.; Madhok, A.A.; Hiasa, H.; Fergusson, D.M. (2006). Synthesis and evaluation of acridine and acridone - based anti - herpes agents with Topo isomerase activity; Bioorganic and Medicinal Chemistry., 14(16), 3467 - 3480.

Gupta, S.K.; Baboo. P. (2015). Synthesis and antimicrobial activity of novel $\mathrm{N}^{10}$-alkyl substituted acridone-9-one derivative. International. J. Research and Development in Pharmacy and life Science., 4(1), 1379-1385.

Hodgemon, D.K.C.; Preger, R.H. (1972). Preparation and properties of the monobromo, nitro, amino and piperdino-10-methylacridones. Aust. J .Chem. 25,191-9.

Huang, C.; Yan, Sh-J.; Zeng, X-h.; Sun, B.; Lan, M-B.; Lin, J. (2015). Synthesis and evaluation of the antitumor activity of polyhalo acridone derivatives. RSC Advances., 5(23),1744417450.

Jameel, R.K.; Mohammad, S.Kh. (2011). Synthesis of some acridine and acridone derivatives. The second scientific conference in chemistry, Coll. Science, Mosul (22-23 November, 2011). 734-746.

Jameel, R.K.; Sheat, A.M. (2014). Synthesis of some new Schiff bases and hydrazones containing benzonaphthyridine / benzonaphthyridine moiety. Raf. J. Sci., 25(3), 56-69.

Kadryavtsera, T.N.; Sysoev, P.I.; Popkov, S.V.; Nazarov, G.V.; Klimova, L.G. (2015). Synthesis and antimicrobial activity of some acridone derivatives bearing 1,3,4-oxadiazole moiety. Russian Chemical Bulletin, International edition., 64(6), 1341-1344.

Kandeel, M.M.; Ali, S.M.; Abdelgawad, M.A.; Abdel-Bakky, M.S.; Mahamed, F.E.A. (2016). Synthesis and cytotoxic activity of acridone substituted with benzimidazole, benzoxazole and benzothiozole. Der. Pharma. Chemica., 8(1), 117-123.

Kannappan, N.; Reddy, B.S.R.; Sen, S.; Nagarajan, R.; Dashute, S. (2009). Synthesis and chemical characterization of quinoline Imine derivatives. J. Appl. Chem. Res., 9,59-68. 
Khanaposhtani, M.M.; Mahdavi, M.; Saeedi, M.; Sabourian, R.; Safavi, M.; Khanavi, M.; Foroumade, A.; Shafiee, Kharzadeh, T. (2015). Design, synthesis, biological evaluation, and docking study of cetylcholinesterase inhibitors: new acridone-1,2,4-oxadiazole-1,2,3triazole hybrids. Chem. Biol. Drug. De., 86,1425-1432.

Mei, X.; August, A.T.; Wolf, C.; (2006). Regioselective copper-catalyzed amination of ochlorobenzoic acid: synthesis and solid state structures of $\mathrm{N}$-aryl anthranilic acid derivatives. J. Org. Chem., 71(1),147-149.

Mohammad shabani, Kh.; Aizi, M.; Aghaei, I.; Jahani, R.; Sharfi, Z.; Shamsei, N. (2016). Design, synthesis pharmacological evalution, and docking study of new acridone-based 1,2,4oxadiazoles as potential anticonvulsant agents. Eur. J. Med. Chem.,112,91-98.

Ugarte, M.M.; Cholewinski, G.; Trzonkowaki, F. (2012). Synthesis and biological activity of novel mycophenolic acid conjugates containing nitro-acridine/ acridone derivatives. Eur. J. Med. Chem., 54,197-201.

Valdes, A.E.C. (2011). Acridine and acridones: old and new structure with antimalarial activity. The open Med. Chem. J., 5, 11-20.

Velingker, V.S.; Dandekar, V.D. (2009). Microwave assisted synthesis and evaluation of anticancer activity of substituted acridone analogues. Res. J. Pharm. And Tech., 2(2), $366-370$.

Wang, Y.; Gao, D.; Chem, Zh.; Li, Sh.; Gao, Ch.; Cao, D.; Liu, H.; Jiang, Y. (2013). Acridone derivative $8 \mathrm{a}$ induces oxidative stress- mediated apoptosis in CCRF-CEM Leukemia cells: Application of metabolomics in mechanistic studies of antitumor agents. Plos One., 8(5), 6372.

Wolf, C.; Mei, X. (2003). Synthesis of conformationally stable 1,8-diarylnaphthalenes: development of new photoiuminescent sensors for ion-selective recognition. J. Am. Chem. Soc., 125, 10651-10658. 